Silvia Corbani

\title{
Propagação de frentes de trincas parcialmente fechadas por flexão cíclica
}

\section{Tese de Doutorado}

Tese apresentada como requisito parcial para obtenção do título de Doutor pelo Programa de Pós-Graduação em Engenharia Civil do Departamento de Engenharia Civil do Centro Técnico Científico da PUC-Rio.

Orientador: Prof. Luiz Fernando C. R. Martha Co-orientadores: Prof. Jaime Tupiassú Pinho de Castro Prof. Antonio Carlos de Oliveira Miranda 


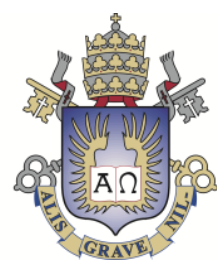

Silvia Corbani

\section{Propagação de frentes de trincas parcialmente fechadas por flexão cíclica}

Tese apresentada como requisito parcial para obtenção do título de Doutor pelo Programa de Pós-Graduação em Engenharia Civil do Departamento de Engenharia Civil do Centro Técnico Científico da PUC-Rio. Aprovada pela Comissão Examinadora abaixo assinada.

Prof. Luiz Fernando C. R. Martha Orientador Departamento de Engenharia Civil - PUC-Rio

Prof. Jaime Tupiassú Pinho de Castro Departamento de Engenharia Mecânica - PUC-Rio Co-orientador

Prof. Antonio Carlos de Oliveira Miranda Universidade de Brasília

Co-orientador

Prof. Carlos Alberto de Almeida Departamento de Engenharia Mecânica - PUC-Rio

Prof. Raul Rosas e Silva Departamento de Engenharia Civil - PUC-Rio

Prof. Gustavo Henrique Bolognesi Donato Centro Universitário da FEI

Prof. Túlio Nogueira Bittencourt

Escola Politécnica da USP

Prof. José Eugênio Leal

Coordenador Setorial do Centro Técnico Científico - PUC-Rio

Rio de Janeiro, 15 de Outubro de 2012 
Todos os direitos reservados. É proibida a reprodução total ou parcial do trabalho sem a autorização da universidade, da autora e do orientador.

\section{Silvia Corbani}

Graduou-se em Engenharia Civil na Escola de Engenharia Mauá (Instituto Mauá de Tecnologia - IMT) em 2001, concluiu mestrado em Engenharia Civil com ênfase em Estruturas pela Universidade de São Paulo em 2006.

Ficha Catalográfica

Corbani, Silvia

Propagação de frentes de trincas parcialmente fechadas por flexão cíclica / Silvia Corbani ; orientador: Luiz Fernando C. R. Martha ; co-orientadores: Antonio Carlos $\mathrm{O}$. Miranda, Jaime Tupiassú P. de Castro - 2012. 236 f. il. (color.) ; $30 \mathrm{~cm}$

Tese (doutorado)-Pontifícia Universidade Católica do Rio de Janeiro, Departamento de Engenharia Civil, 2012.

Inclui bibliografia

1. Engenharia civil - Teses. 2. Fadiga. 3. Propagação de trincas. 4. Trincas de superfície. 5. Método dos elementos finitos. I. Martha, Luiz Fernando C. R. II. Miranda, Antonio Carlos O. III. Castro, Jaime Tupiassú P. de. IV. Pontifícia Universidade Católica do Rio de Janeiro. Departamento de Engenharia Civil. III. Título. 
À memória de

Vanda Ciampi Corbani. 


\section{Agradecimentos}

Este trabalho não poderia ter sido feito sem a ajuda do orientador e coorientadores: Luiz Fernando Martha, Antonio Carlos Miranda e, principalmente, Jaime Tupiassú de Castro. Agradeço a todos pela paciência, recomendações e explicações, guiando este trabalho para um término bem-sucedido.

Ao professor Anthony Ingraffea, pelas explicações durante o período em Cornell. Ao Bruce Carter, por todas as adaptações feitas no FRANC3D. Aos professores Topper, Ivan Menezes e Ronaldo Vieira, pelas dicas e explicações. Aos professores Gustavo Donato e Marco Antonio Meggiolaro, pelas sugestões e correções. Aos professores José Alexander Araújo e Jorge Luiz Ferreira, ao aluno Marcus Sá, pelos ensaios da/dN padronizados executados na UNB.

Aos profissionais da PUC-Rio, especialmente, Heitor, Euclides, José e Rita.

Ao Theophilo Trabulsi Filho e sua empresa, pela doação dos perfis metálicos.

Aos queridos amigos Marquito, Gerardo, Cri-cri, Biruta, Leo e Jaiminho, por fazerem o dia-a-dia no laboratório ser mais leve e divertido. Em especial, ao Gerardo, pela montagem e desmontagem do suporte. Ao Albert Cerroni, pela amizade em Cornell.

Ao marido, André, pela motivação e incentivo. A minha mãe (in memorian) e meu pai, Vanda e Estevão. Aos ensinamentos budistas. Às companheiras de prática, Vera, Ana Paiva e Flavia.

Ao CNPq pela concessão de bolsa no Brasil e bolsa-sanduíche, sem a qual não conseguiria ter me dedicado exclusivamente a esta pesquisa. 


\section{Resumo}

Corbani, Silvia; Martha, Luiz Fernando C. R. Propagação de frentes de trincas parcialmente fechadas por flexão cíclica. Rio de Janeiro, 2012. 236p. Tese de Doutorado - Departamento de Engenharia Civil, Pontifícia Universidade Católica do Rio de Janeiro.

Neste trabalho são investigadas experimentalmente e numericamente as mudanças de geometria em trincas inicialmente passantes submetidas a carregamento remoto de flexão pura induzindo fechamento parcial das faces da trinca. Esse crescimento de trinca pode ocorrer numa variedade de estruturas com defeitos pré-existentes, tais como fuselagens de aviões, cascos de navios, vasos de pressões e pontes metálicas. O carregamento de flexão pura ocasiona regiões de tração e compressão na frente da trinca. É inquestionável que parte das faces da trinca sob compressão fecha independentemente de qualquer mecanismo de fechamento; e outra parte das faces da trinca, por outro lado, sob tração cresce mudando gradualmente de geometria. Após realizar ensaios em corpos-de-prova de aço ASTM A-36, foi observado que tais carregamentos geram uma quina na frente da trinca, que é a transição de uma geometria parcialmente passante e um trecho remanescente da geometria inicial. Para entender a distribuição do fator de intensidade de tensão em tais frentes de trinca, suas geometrias foram reproduzidas em um modelador tridimensional de mecânica da fratura linear elástica, o FRANC3D, acoplado a um programa de análise de elementos finitos (ABAQUS). Com este sistema acoplado, foram executadas análises considerando efeitos não lineares causados pelo contato das faces da trinca sob compressão. Verificou-se a necessidade de propor metodologias para tratamento dos resultados numéricos na quina, obtendo-se predições eficientes das mudanças na geometria da trinca. Contudo, a estimativa de vida, quando se compara taxas de crescimento da trinca obtidas em um corpo-de-prova sob tração cíclica e as taxas em um corpo-de-prova sob flexão com fechamento parcial da trinca, foi melhor reproduzida usando um fator de correção de fechamento da trinca. Adicionalmente, uma série de expressões empíricas normalizadas para geometrias da trinca e fatores de intensidade de tensão são propostas.

\section{Palavras-chave}

Fadiga; Propagação de trincas; Trincas de superfície; Método dos elementos finitos. 


\section{Abstract}

Corbani, Silvia; Martha, Luiz Fernando C. R. Crack growth with partial bending-induced crack closure. Rio de Janeiro, 2012. 236p. DSc. Thesis Departamento de Engenharia Civil, Pontifícia Universidade Católica do Rio de Janeiro.

This work investigates experimentally and numerically how the front of initially through edge cracks in plate changes after they pass to be remotely fatigue loaded under pure bending to induce partial closure of the crack faces. This type of crack growth problem can occur in a variety of structures with preexisting defects, such as aircraft fuselages, ship hulls, pressure vessels components, and steel bridges. The bending loads induce tension and compression regions along the crack front, with the part of the crack faces that work under compression undoubtedly closed by the load, independently of any other closure mechanism. The part of the crack faces that work under tension; on the other hand, crack grows by fatigue gradually changing its shape. After performing tests on ASTM A36 steel specimens, it was observed that the bending load induces a kink on the crack front, in the transition between the part through crack created on the tension side and initial crack geometry. To understand the distribution of the stress intensity factor along such crack fronts, the measured crack shapes were reproduced in a three-dimensional fracture mechanics modeler (FRANC3D) coupled to a finite element analysis program (ABAQUS). With this coupled system, linear elastic stress analysis simulations were performed considering the nonlinear effects caused by the crack face contact in the compressed region. In particular, methods had to be proposed to treat numerical noise around the kink. The proposed methodology efficiently predicts the observed crack front shape changes; although the observed fatigue lives were better reproduced using a crackclosure correction factor when compared to crack growth data obtained from standard compact tension specimens. In addition, a series of normalized empiric expressions for both crack front shapes and stress intensity factors are proposed.

\section{Keywords}

Fatigue; Crack growth; Surface crack; Finite element. 


\section{Sumário}

1 Introdução 28

1.1. Problema 28

1.2. Motivação 31

1.3. Revisão bibliográfica 32

1.3.1. Propagação em trincas de superfície 33

1.3.2. Contato parcial das faces da trinca sob flexão 36

1.4. Contribuições e objetivos 37

1.5. Organização do trabalho 39

2 Estimativas do fator de intensidade de tensão 41

2.1. Modos de fratura de teoria de elasticidade tri-dimensional 41

2.2. Tensões e deslocamentos em uma placa trincada infinita 42

2.3. Trincas passantes e trincas de superfícies 45

2.4. FIT em peças com dimensões finitas em trincas retas e passantes 48

2.5. FIT em trincas bidimensionais 48

2.6. Cálculo numérico de FIT em problemas 3D 50

2.6.1. Tensões singulares em elementos finitos 51

2.6.2. Método da integral - $M$

2.6.3. Método de correlação de deslocamentos 57

3 Propagação da trinca por fadiga $\quad 59$

3.1. Carregamento de fadiga $\quad 59$

3.2. Conceito e aplicação de similaridade 60

3.3. Equações empíricas de crescimento da trinca por fadiga 62

3.4. Fechamento da trinca, $\Delta K_{\text {eff }} \quad 64$

3.5. Propagação da trinca de superfície 66

3.5.1. Critério de incremento da trinca 67

3.5.2. Direção de propagação 68

3.6. Determinação do número de ciclos 70

4 Procedimentos e resultados experimentais 72 
4.1. Corpos-de-prova (CPs) 72

4.2. Suportes para o ensaio de flexão 76

4.2.1. Motivação 76

4.2.2. Novos acessórios $\quad 78$

4.3. Propriedades do material $\quad 81$

4.4. Descrição do experimento 82

4.5. Aquisição de dados 86

4.5.1. Medidas do comprimento da trinca 86

4.5.2. Blocos de carregamentos 90

4.5.3. Medidas de deformações 92

4.6. Resultados experimentais 93

4.6.1. CP com entalhe de borda 94

A. Carregamento de fadiga com $\Delta K$ constante $\quad 94$

B. Blocos de sobrecarga 99

C. Blocos de subcargas 101

4.6.2. CP com entalhe central 104

4.7. Avaliação e resumo dos dados coletados 105

4.8. Simplificação da geometria no plano de trincamento 106

5 Análise numérica dos parâmetros usados na reconstrução do crescimento da trinca 119

5.1. Descrição do problema e hipóteses usadas 120

5.2. Singularidades numéricas na frente da trinca 122

5.3. Metodologias usadas para tratar a quina 126

5.3.1. Suavização da frente da trinca 126

5.3.2. Extrapolação de $\Delta a \quad 128$

5.3.3. Extrapolação de $\Delta K_{l} \quad 129$

5.4. Direção do crescimento da trinca 132

5.5. Sensibilidade da geometria da trinca a $d a / d N-\Delta K$ e a $\Delta K_{\operatorname{máx}} \quad 136$

5.6. Simplificação da geometria da trinca como quarto-elipses 139

6 Predição numérica do crescimento da trinca 142

6.1. Ajustes da taxa de crescimento da trinca 142

6.2. Predição das geometrias da trinca durante seu crescimento 144

6.3. Discussão dos resultados 151 
6.4. Previsão de vida 152

6.5. Hipótese de direção de crescimento da trinca 154

7 Avaliação de $\Delta K$ nas geometrias dos experimentos 156

7.1. Frentes de trincas marcadas por aumentos de cargas 156

7.2. Frentes de trincas marcadas por blocos de sobrecargas 160

7.3. Frentes de trincas marcadas por blocos de subcargas 163

7.4. Funções de geometria para o CP com um entalhe de borda 168

$\begin{array}{ll}\text { 7.4.1. Solução de } \Delta K_{l, c} & 169\end{array}$

$\begin{array}{ll}\text { 7.4.2. Solução de } \Delta K_{l, a} & 170\end{array}$

$\begin{array}{ll}\text { 7.4.3. Solução de } \Delta K_{/} \text {no CP01 } & 171\end{array}$

$\begin{array}{ll}\text { 7.5. Estimativa da vida à fadiga no CP01 } & 174\end{array}$

$\begin{array}{ll}\text { 7.5.1. Valores aceitáveis } & 175\end{array}$

$\begin{array}{ll}\text { 7.5.2. Curvas } d a / d N-\Delta K & 175\end{array}$

7.5.3. $\Delta \mathrm{K}$ na superfície inferior da placa e na profundidade da trinca 176

7.5.4. Comparação das taxas de propagação 178

$\begin{array}{ll}\text { 7.5.5. Previsão de vida à fadiga } & 183\end{array}$

7.5.6. Correção do fator de intensidade de tensão 186

8. Comentários e conclusões 193

8.1. Principais contribuições 193

$\begin{array}{lr}\text { 8.2. Sugestões para futuros trabalhos } & 197\end{array}$

$\begin{array}{lr}\text { Referências bibliográficas } & 198\end{array}$

Apêndice 1 - Teste de convergência 203

Apêndice 2 - Ajuste de ensaios $d a / d N-\Delta K \quad 209$

Apêndice 3 - Projeto dos corpos-de-prova para pré-trinca 217

Apêndice 4 - Resumo dos resultados experimentais 223

Apêndice 5 - Tabelas dos carregamentos aplicados 227

Apêndice 6 - Considerações do contato 230

Apêndice 7 - Comparação das funções de geometria na interseção da trinca com a superfície inferior da placa 234 


\section{Lista de figuras}

Figura 1.1 - Geometria da frente da trinca sob tração em uma placa finita:

(a) trinca curta e (b) trinca longa.

Figura 1.2 - Problema de propagação de trinca submetida à flexão pura.

Figura 1.3 - Resultados de FIT sob carregamento de flexão:

(a) Geometria da trinca de superfície e (b) Resultados típicos de FIT (Newman \& Raju, 1981).

Figura 2.1 - Três modos principais de fratura: (a) Modo I, modo de abertura;

(b) Modo II, modo de cisalhamento no plano e (c) Modo III, modo de rasgamento.

Figura 2.2 - Parâmetros usados para descrever as tensões na ponta da trinca. $\quad 43$

Figura 2.3 - Carregamento remoto aplicado para os três modos de fratura. $\quad 45$

Figura 2.4 - Diferentes geometrias de frente de trinca AB para trincas

passante e trincas parcialmente passantes (Schijve, 2009).

Figura 2.5 - Nomenclatura da trinca em problemas bidimensionais:

(a) trinca de borda e (b) trinca central.

Figura 2.6 - Nomenclatura da trinca em problemas tridimensionais

utilizando a geometria dos corpos-de-prova ensaiados:

(a) trinca de borda e (b) trinca central.

Figura 2.7 - Parâmetros usados na determinação do FIT na geometria de trinca de canto.

Figura 2.8 - Fator de intensidade de tensão na trinca curva:

(a) $K_{I, c}$; e (b) $K_{I, a}$.

Figura $2.9-K_{I}$ ao longo de toda a frente da trinca.

Figura 2.10 - Roseta: (a) seção transversal em problemas bidimensional e tridimensional e (b) trajetória da roseta em um problema tridimensional.

Figura 2.11 - Ponta da trinca em problemas 2D: (a) uma curva arbitrária e (b) uma área arbitrária $A$. 
Figura 2.12 - Numeração do elemento e sistema de coordenadas:

(a) Elemento quarter-point C3D15 em sistema de coordenadas cartesiano; e (b) Disposição dos elementos e convenção dos nós.

Figura 3.1 - Histórico do carregamento aplicado.

Figura 3.2 - Predição do crescimento da trinca em um conector com trinca passante.

Figura 3.3 - Três regiões da taxa de crescimento da trinca como função de $\Delta K$ (Schijve, 2009).

Figura 3.4 - Predição de taxa de crescimento de trinca de fadiga: (a)

Resultados de predição e ensaio; (b) Dados do material e (c) $\Delta K_{\text {eff }}$ calculado. 65

Figura 3.5 - Histórico do carregamento de tensões (Schijve, 2009).

Figura 3.6 - Crescimento da trinca: (a) Distribuição de $\Delta K$ ao longo

da frente da trinca e (b) Distribuição dos incrementos na frente da trinca.

Figura 3.7 - Direção de propagação perpendicular a toda frente de trinca atual.

Figura 3.8 - Índices para cálculo de número de ciclos em trinca de superfície com fechamento parcial.

Figura 4.1 - Geometria dos CPs com dimensões em milímetros:

(a) um entalhe central e (b) um entalhe de borda.

Figura 4.2 - Esquema das configurações das placas com dimensões em milímetros: (a) um entalhe central e (b) um entalhe de borda.

Figura 4.3 - Acessórios (a) ensaio em três pontos e (b) ensaio em quatro pontos.

Figura 4.4 - Superfície da trinca de fadiga, usando o aparato ilustrado na Figura 4.3 (b).

Figura 4.5 - Configuração esquemática do aparato de flexão com dimensões em milímetros.

Figura 4.6 - Guia de ligação da base do apoio com perfil metálico e guia em L para acomodar o CP.

Figura 4.7 - Guias em L evitaram giro da parte inferior do aparato de flexão. $\quad 80$

Figura 4.8 - Esbarros nas extremidades do CP.

Figura 4.9 - Experimento de pré-trincamento: (a) um entalhe de borda,

(b) um entalhe central. 
Figura 4.10 - Experimento: (a) Aparatos de flexão e CP;

(b) Vista do experimento.

Figura 4.11 - Aquisições do comprimento da trinca no ponto A e B:

(a) configuração inicial; (b) durante ensaio.

Figura 4.12 - Esquema do aparato de medição da frente da trinca

(a) micrômetro analógico e (b) micrômetro digital.

Figura 4.13 - Marca usada para medir incrementos de trinca. 89

Figura 4.14 - Microscópios USB usados no experimento. 90

Figura 4.15 - Crescimento da trinca por bandas (a) Carregamento

de fadiga e; (b) superfície da trinca.

Figura 4.16 - Strain-gages colados: (a) na superfície superior e

(b) na superfície inferior.

Figura 4.17 - Aquisição de dados: (a) Strain Indicator e Recorder Vishay

Model P3, (b) tela LCD. 93

Figura 4.18 - Histórico das tensões aplicadas na superfície inferior da placa. 95

Figura 4.19 - Trinca de superfície no CP01. 95

Figura 4.20 - Trinca de fadiga no CP01: (a) Microscópio óptico;

(b) Câmera digital; (c) Legenda.

Figura 4.21 - Largura de colaboração na placa: (a) sob carregamento remoto de compressão e (b) sob carregamento remoto de tração. 98

Figura 4.22 - Histórico das tensões aplicadas na superfície inferior da placa. 99

Figura 4.23 - Superfície da trinca de fadiga do CP06: (a) e (b) Microscópio

óptico; (c) Legenda.

100

Figura 4.24 - Quinas na frente da trinca.

100

Figura 4.25 - Regiões com deformação plástica na superfície superior da placa.

Figura 4.26 - Histórico das tensões normais aplicadas na superfície inferior da placa: CP07.

Figura 4.27 - Trinca de superfície no CP07 (a) foto com microscópio Zeiss; (b) e (c) fotos com máquina digital.

Figura 4.28 - Legenda na trinca de superfície para diversas marcas de frente de trinca.

Figura 4.29 - Ângulo de interseção da frente da trinca com superfície livre. 104

Figura 4.30 - Trinca de superfície CP03: (a) trinca A; (b) trinca B. 
Figura 4.31 - Trinca de superfície CP02 no microscópio Zeiss:

(a) trinca A; (b) trinca B. 104

Figura 4.32 - Curva $d$ vs. $N$ dos CPs com entalhe de borda. 105

Figura 4.33 - Comparação da frente da trinca no CP01

com quarto-elípticas concêntricas.

Figura 4.34 - Comparação da frente da trinca no CP06

com quarto-elípticas concêntricas.

Figura 4.35 - Comparação da frente da trinca do CP07

com quarto-elípticas concêntricas.

Figura 4.36 - Coordenadas do centro $X$ no CP01 das curvas

quarto-elípticas concêntricas.

Figura 4.37 - Coordenada do centro $X$ no CP06 das curvas

quarto-elípticas concêntricas.

Figura 4.38 - Coordenadas do centro $X$ no CP07 das curvas

quarto-elípticas concêntricas.

Figura 4.39 - Comparação entre frente de trinca experimental

e um quarto de elipses não concêntricas no CP01.

Figura 4.40 - Comparação entre marcas de experimento e curvas

quarto-elípticas não concêntricas no CP06.

Figura 4.41 - Comparação entre marcas de experimento e curvas

quarto-elípticas não concêntricas no CP07.

Figura 4.42 - Coordenadas na frente da trinca: (a) Indicação de corte $A-A$

na placa; (b) Corte $A-A$ trinca curta; (c) Corte $A-A$ trinca longa.

Figura 4.43 - Curvas de ajuste de $a / t$ e centro $x_{c}$.

Figura 4.44 - Esboço da trinca de superfície para $a / t=1$.

Figura 4.45 - Comparação da frente de trinca obtida no CP03 com

quarto-elipses: (a) trinca A e (b) trinca B.

Figura 4.46 - Comparação da frente de trinca obtida no CP02 com quarto-elipses: (a) trinca A e (b) trinca B.

Figura 5.1 - Descrição do problema estudado numericamente:

(a) Esquema da placa tridimensional e carregamento aplicado de flexão pura e (b) Indicação da região de contato e trecho da frente da trinca que cresce na seção de ligamento. 
Figura 5.2 - Esquema da geometria da frente da trinca: (a) Localização da quina obtida nos corpos-de-prova ensaiados e (b) raio de arredondamento utilizado para gerar a geometria do ensaio no FRANC3D.

Figura 5.3 - Distribuição do $\Delta K_{I}$ usando diversas geometrias de trincas obtidas nos corpos-de-prova ensaiados.

Figura 5.4 - Distribuição do FIT na frente da trinca normalizada usando a geometria da Marca 03b no CP07 com diversos raios de arredondamento e sob carregamento remoto de flexão pura ou sob tração.

Figura 5.5 - Fluxograma das etapas da simulação do crescimento da trinca incorporado ao programa FRANC3D.

Figura 5.6 - Esquema da geometria da frente de trinca atual e curva obtida pela metodologia de extrapolação de , preservando a quina na geometria da trinca subsequente.

Figura 5.7 - Geometrias das frentes da trinca atual e trinca subsequente usando a metodologia de extrapolação de $\Delta a_{i}$.

Figura 5.8 - Identificação dos trechos importantes na distribuição do FIT usando marcas de frente da trinca obtidas nos corpos-de-prova ensaiados. Figura 5.9 - Coordenadas na frente da trinca usando geometria simplificada: (a) em trinca curta e (b) em trinca longa.

Figura 5.10 - Hipótese da direção de crescimento com vetor normal no plano da seção de ligamento: (a) em uma trinca reta passante sob tração remota e (b) em uma trinca de superfície sob tração e/ou flexão remota. Figura 5.11 - Distribuição das tensões normais obtida no ABAQUS na seção de ligamento usando uma geometria de trinca usando uma geometria de trinca extraída dos corpos-de-prova ensaiados: (a) sob carregamento remoto de tração simples e (b) sob carregamento remoto de flexão pura. Figura 5.12 - Esquema da direção de crescimento na frente de trinca usando a geometria dos corpos-de-prova ensaiados sob carregamento remoto de tração simples.

Figura 5.13 - Esquema de uma hipótese mais geral da direção de crescimento na frente de trinca usando a geometria dos corpos-de-prova ensaiados sob carregamento remoto de flexão pura.

Figura 5.14 - Comparação da geometria da frente de trinca subsequente determinada com lei de propagação ajustada por Hall e lei de Paris. 
Figura 5.15 - Comparação da geometria da frente de trinca subsequente na quina usando ajustes da lei de propagação com Lei de Paris e com ajuste de Hall para diversos incrementos máximos de trinca.

Figura 5.16 - Aproximação das frentes de trinca intermediárias entre Marca 02 e Marca 03 do CP01 por curvas quarto-elípticas concêntricas.

Figura 5.17 - Legenda usada nas frentes de trinca analisadas no FRANC3D. 140 Figura 5.18 - Distribuição do FIT normalizado determinado no FRANC3D, usando geometrias obtidas no corpo-de-prova CP01 e geometrias simplificadas por curvas quarto-elípticas concêntricas.

Figura 6.1 - Curva $d a / d N-\Delta K$ usadas na predição do crescimento do CP01.

Figura 6.2 - Distribuição do FIT no passo 32 com a geometria da frente da trinca obtida incrementalmente no FRANC3D.

Figura 6.3 - Distribuição do FIT no passo 89 com a geometria da frente da trinca obtida incrementalmente no FRANC3D.

Figura 6.4 - Comparação da geometria obtida na predição numérica com a geometria do CP01.

Figura 6.5 - Predições de geometria obtidas no crescimento com $d a / d N-\Delta K$ de um corpo-de-prova sob tração: (a) Geometria da frente da trinca a cada 8 passos e (b) Legenda para relacionar a frente da trinca com a distribuição do FIT na figura a seguir.

Figura 6.6 - Distribuição do FIT normalizado ao longo do ângulo $\phi$ obtidos no crescimento da trinca com a $d a / d N-\Delta K$.

Figura 6.7 - Predições de geometria obtidas no crescimento com $d d / d N-\Delta K$ do CP01: (a) Geometria da frente da trinca a cada 8 passos e (b) Legenda para relacionar a frente da trinca com a distribuição do FIT na figura a seguir.

Figura 6.8 - Comparação das geometrias obtidas incrementalmente com a geometria do CP01.

Figura 6.9 - Distribuição do FIT normalizado ao longo do ângulo $\phi$ obtidos no crescimento da trinca com a $d d / d N-\Delta K$.

Figura 6.10 - Desvio da geometria da trinca.

Figura 6.11 - Soluções discretas de fator de forma obtidas nas análises de EF. 
Figura 6.12 - Estimativas de vida na superfície inferior da placa.

Figura 6.13 - Distribuição do FIT na superfície de trincamento com geometria da pré-trinca.

Figura 6.14 - Direção de propagação: (a) Passo 1 e (b) Passo 2.

Figura 7.1 - Marcas das geometrias da trinca no CP01:

(a) Corpo-de-prova e (b) Geometria das frentes de trinca inseridas no programa FRANC3D.

Figura 7.2 - FIT normalizado em relação ao ângulo $\phi$ obtido no FRANC3D com as marcas do CP01.

Figura 7.3 - Extrapolações do FIT normalizado no CP01, usando metodologia de extrapolação do FIT.

Figura 7.4 - Marcas das geometrias da trinca no CP06: (a) Corpo-de-prova e (b) Geometria da trinca usada no FRANC3D.

Figura 7.5 - FIT normalizado em relação ao ângulo obtido $\phi$ no FRANC3D com as marcas do CP06.

Figura 7.6 - Extrapolações do FIT normalizado para CP06, usando metodologia de extrapolação do FIT.

Figura 7.7 - Marcas das geometrias da trinca no CP07: (a) Corpo-de-prova e (b) Geometria da marcas de frente da trinca usada na Figura 7.8.

Figura 7.8 - FIT normalizado em relação ao ângulo $\phi$ obtido no FRANC3D com as marcas do CP07.

Figura 7.9 - Extrapolações do FIT normalizado no CP07, usando metodologia de extrapolação do FIT.

Figura 7.10 - Parâmetros usados na determinação do FIT na geometria de trinca de canto.

Figura 7.11 - Pontos discretos de $F$ em $C$ e polinômio da função de geometria utilizada em $\Delta K_{I, c}$.

Figura 7.12 - Pontos discretos de $F$ em $A$ e polinômio da função de geometria utilizada em $\Delta K_{I, a}$.

Figura 7.13 - Marcas das frentes da trinca obtidas no corpo-de-prova CP01, ilustrando a distância entre marca 02 e 03. 
Figura 7.14 - Marcas obtidas nos corpos-de-prova CP01 e CP07, com distribuições $\Delta K_{I}$ determinadas no FRANC3D aproximadas por uma solução geral de interpolação destas marcas.

Figura 7.15 - Fator de intensidade de tensão em $C$ no CP01.

Figura 7.16 - Fator de intensidade de tensão em $A$ no CP01.

Figura 7.17 - Trajetórias da trinca adotada na superfície inferior da placa e trajetória 3.

Figura 7.18 - Ajuste polinomial dos dados $d v s . N$ coletados durante ensaio no CP01.

Figura 7.19 - Comparação das taxas de propagação no corpo-de-prova padrão sob tração (trinca passante) e taxas de propagação no CP01 (trinca de superfície), localizadas em A e em C.

Figura 7.20 - Trajetórias de crescimento da trinca partindo da marca 01, usando direção de crescimento normal a marca atual.

Figura 7.21 - Determinação do número de ciclos acumulados nas marcas do CP07 eliminando retardos ou acelerações.

Figura 7.22 - Taxas de propagação ao longo das trajetórias 1, 2 e 3.

Figura 7.23 - Comparação de $d v s$. $N$ com previsão numérica da vida usando $\Delta K_{I, c}$ e medidas do ensaio no CP01.

Figura 7.24 - Comparação de $a$ vs. $N$ com previsão numérica da vida usando $\Delta K_{I, a}$ e medidas do ensaio no CP01.

Figura 7.25 - Taxas de crescimento do aço ASTM A36 ajustadas com lei de Paris $d a / d N-\Delta K_{I}$ e $d a / d N-\Delta K_{\text {eff }}$.

Figura 7.26 - Gráfico $K_{\max } v s . K_{o p}$ em $A$ e em $C$.

Figura 7.27 - Gráfico $U_{\text {sup }} v s . \Delta K_{I, \text { sup }}$ em $A$ e em $C$.

Figura 7.28 - Predição de vida usando $\Delta K_{\text {eff }}$ em $C$.

Figura 7.29 - Predição de vida usando $\Delta K_{\text {eff }}$ em $A$.

Figura A1. 1 - Dados para análise: (a) Dimensões da placa trincada [mm] e;

(b) Malha na região da trinca e demais regiões e condições de contorno.

Figura A1. 2 - Dimensões dos elementos nas regiões de malha sem trinca.

Figura A1. 3 - Deslocamento no nó $A$ para diversas malhas nas regiões sem trinca.

Figura A1. 4 - FIT ao longo da frente de trinca normalizada. 
Figura A1. 5 - Roseta na ponta da trinca: (a) três anéis de elementos e (b) cinco anéis de elementos.

Figura A1. 6 - Refinamento dos elementos na frente da trinca: (a) FIT na frente da trinca e (b) FIT na quina.

Figura A2. 1 - Geometria do corpo-de-prova padrão compacto de tração $\mathrm{C}(\mathrm{T})$.

Figura A2. 2 - Primeira amostra de dados coletados, ensaio de taxa de propagação de trinca, usando um $\mathrm{C}(\mathrm{T})$.

Figura A2. 3 - Pontos medidos na segunda amostra de dados coletados, usando $\mathrm{CP}$ compacto $\mathrm{C}(\mathrm{T})$.

Figura A2. 4 - Pontos para ajuste de curva.

Figura A2 .5 - Ajuste de curva nos pontos médios.

Figura A2. 6 - Ajuste de curva Hall 4 parâmetros nos pontos superiores. 214

Figura A2. 7 - Ajuste de curva Hall 4 parâmetros nos pontos inferiores.

Figura A2. 8 - Comparação da curva medida com resultados disponíveis na literatura.

Figura A3. 1 - Geometria do corpo-de-prova em [mm]:

(a) entalhe de borda; (b) entalhe central e (c) dois entalhes de borda.

Figura A3. $2-f(a / w)$ para corpo-de-prova com entalhe de borda.

Figura A3. $3-f(a / w)$ para corpo-de-prova com entalhe central.

Figura A3. $4-f(a / w)$ para corpo-de-prova com dois entalhes de borda.

Figura A3. 5 - Trinca no furo do CP05.

Figura A6. 1 - Penetração da superfície mestre na superfície escrava sob contato mestre-escrava com uma discretização grosseira da malha, atendendo as condições de compatibilidade nó em superfície (Hibbitt, 1996).

Figura A6. 2 - Contato em um sólido elástico.

Figura A6. 3 - Relação pressão-fechamento (Hibbitt, 1996).

Figura A7. 1 - Geometria da frente da trinca: (a) trinca do experimento; (b) trinca reta-passante.

Figura A7. 2 - Comparação da função de geometria em $C-f(d / w)$. 


\section{Lista de tabelas}

Tabela 4.1 - As dimensões exatas dos CPs, condições iniciais:

(a) entalhe central; e (b) entalhe de borda.

Tabela 4.2 - Propriedades de engenharia do material aço ASTM A36.

Tabela 4.3 - Tipo de histórico de carregamento, número de ciclos total $\mathrm{Ne}$ razão final $a / w$ : (a) entalhe central; (b) entalhe de borda.

Tabela 4.4 - Semi-eixos das curvas quarto-elipses concêntricas no CP01, usadas na Figura 4.33.

Tabela 4.5 - Semi-eixos das curvas quarto-elipses concêntricas no CP06, usadas na Figura 4.34.

Tabela 4.6 - Semi-eixos das curvas quarto-elipses concêntricas no CP07, usadas na Figura 4.35.

Tabela 4.7 - Comprimento máximo $d$ da trinca simplificada apenas por curva quarto-elíptica.

Tabela 4.8 - Novo centro e semi-eixos da Marca03 do CP01, ilustradas na Figura 4.39.

Tabela 4.9 - Novo centro e semi-eixos de 3 Marcas no CP06, ilustradas na Figura 4.40.

Tabela 4.10 - Novos centros e semi-eixos de 12 Marcas no CP07, conforme ilustrado na Figura 4.41.

Tabela 4.11 - Resumo das coordenadas de centro nos três CPs.

Tabela 4.12 - Semi-eixos das elipses concêntricas no CP03.

Tabela 4.13 - Semi-eixos das elipses concêntricas no CP02.

Tabela 6.1 - Comparação da geometria da trinca na predição numérica e geometria do CP01.

Tabela 6.2 - Coordenadas da geometria da trinca do CP01 e comprimento $O P$.

Tabela 6.3 - Resumo das estimativas totais de número de ciclos em $d$.

Tabela 7.1 - Fatores de geometria do CP01 determinados no trecho confiável de $\Delta K_{I}$. 
Tabela 7.2 - Fatores de geometria do CP06 determinados no trecho confiável de $\Delta K_{I}$.

Tabela 7.3 - Fatores de geometria do CP07 determinados no trecho confiável de $\Delta K_{I}$.

Tabela 7.4 - Fatores de geometria utilizados na solução geral do FIT, onde $c$ é o semi-eixo da elipse.

Tabela 7.5 - Ângulo $\phi$ [em graus] determinado nas trajetórias usadas para determinar as taxas de crescimento na trinca de superfície.

Tabela 7.6 - Resumo da geometria da trinca nas marcas usadas na expressão de $\Delta K_{I}$.

Tabela 7.7 - Resumo das estimativas totais do número de ciclos $N$ usando expressão de $\Delta K_{I, c}$ e taxas de propagação de um corpo-de-prova padrão sob tração.

Tabela 7.8 - Resumo das estimativas totais de número de ciclos usando expressão de $\Delta K_{I, a}$ e diversos ajustes das taxas de propagação realizadas em um ensaio de tração.

Tabela 7.9 - Dados usados para determinar $U_{\text {sup }}$ na interseção de trinca com a superfície inferior.

Tabela 7.10 - Dados usados para determinar $U_{\text {sup }}$ em $A$.

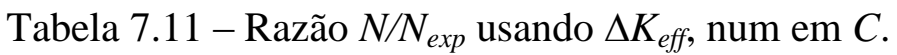

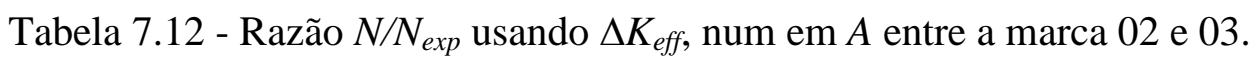

192

Tabela A1. 1 - Dimensões da malha na região sem trinca e resultados.

Tabela A1. 2 - Refinamento da malha na frente da trinca.

Tabela A3. 1 - Carregamento aplicado.

Tabela A3. 2 - Dados do experimento para corpo-de-prova com um entalhe de borda.

Tabela A3. 3 - Dados do experimento para corpo-de-prova com um entalhe central.

Tabela A4. 1 - As dimensões exatas dos CPs, condições iniciais:

(a) entalhe central; e (b) entalhe de borda.

Tabela A4. 2 - Tipo de histórico de carregamento, número de ciclos total $N_{\text {total }}$ e razão final $a / w$ : (a) entalhe central; (b) entalhe de borda. 
Tabela A4. 3 - Resumo das coordenadas de centro (concêntrico) nos CPs com entalhe de borda.

Tabela A4. 4 - Resumo dos dados - CP01. 224

Tabela A4. 5 - Semi-eixos não concêntricos da Marca03 do CP01. 224

Tabela A4. 6-Comprimentos dos semi-eixos das elipses no CP06. 224

Tabela A4. 7 - Novo centro e semi-eixos de 3 Marcas no CP06. 224

Tabela A4. 8 - Comprimentos dos semi-eixos das elipses no CP07. 225

Tabela A4. 9 - Novos centros e semi-eixos no CP07. 226

Tabela A5. 1 - Condições gerais do carregamento de fadiga aplicado $\begin{array}{ll}\text { no CP01. } & 227\end{array}$

Tabela A5. 2 - Histórico de carregamento no CP06. 228

Tabela A5. 3 - Histórico de carregamento no CP07. 229

Tabela A7. 1 - Diferenças na magnitude da função de geometria em diversas razões $d / w$ quando comparadas a função de geometria obtida com a geometria dos corpos-de-prova. 


\section{Lista de abreviaturas}

ASTM - American Society for testing and materials.

$\mathrm{CP}$ - corpo-de-prova.

$\mathrm{EF}-$ elementos finitos.

FIT - Fator de intensidade de tensão.

MFLE - Mecânica da fratura linear elástica. 


\section{Lista de símbolos}

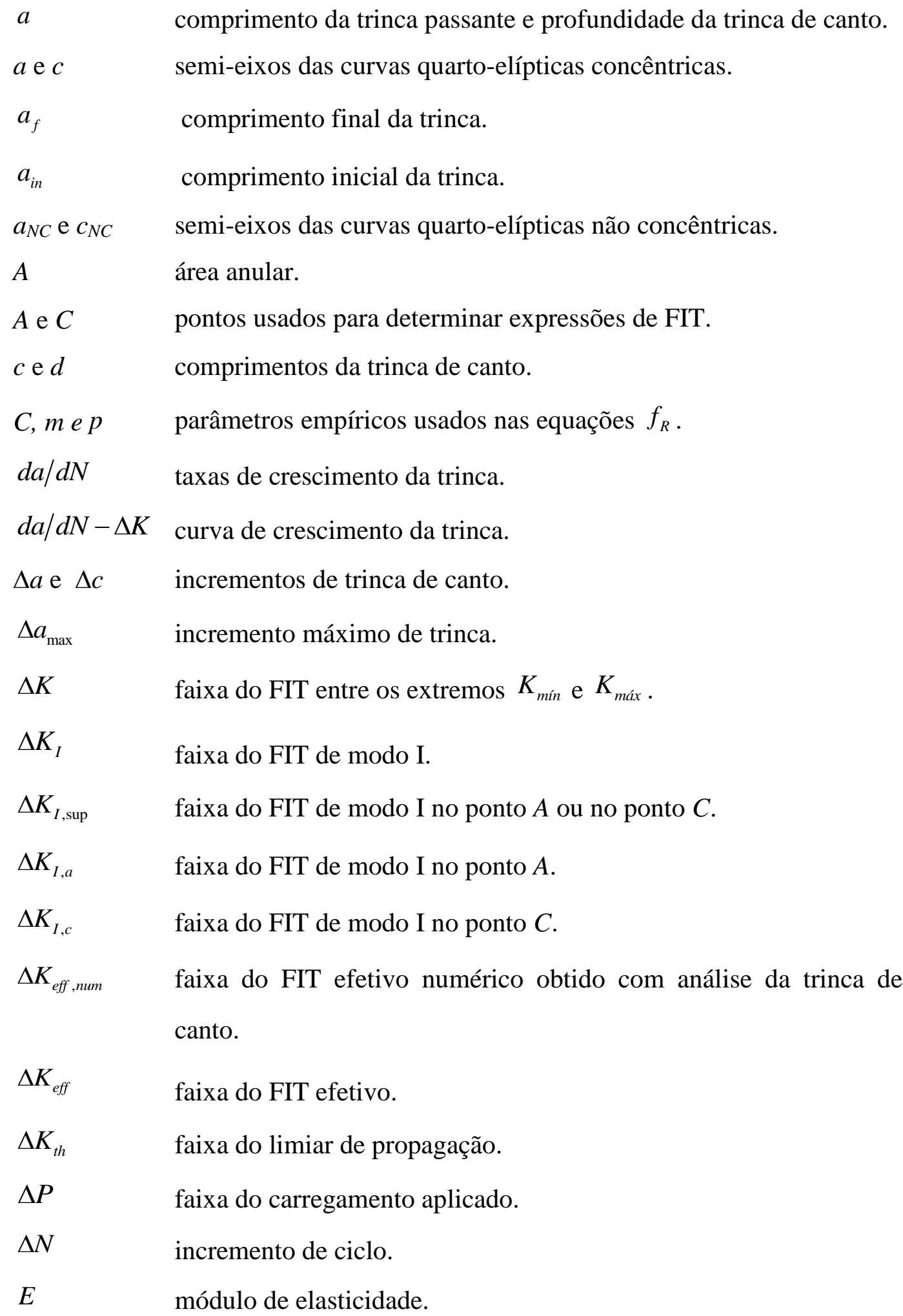




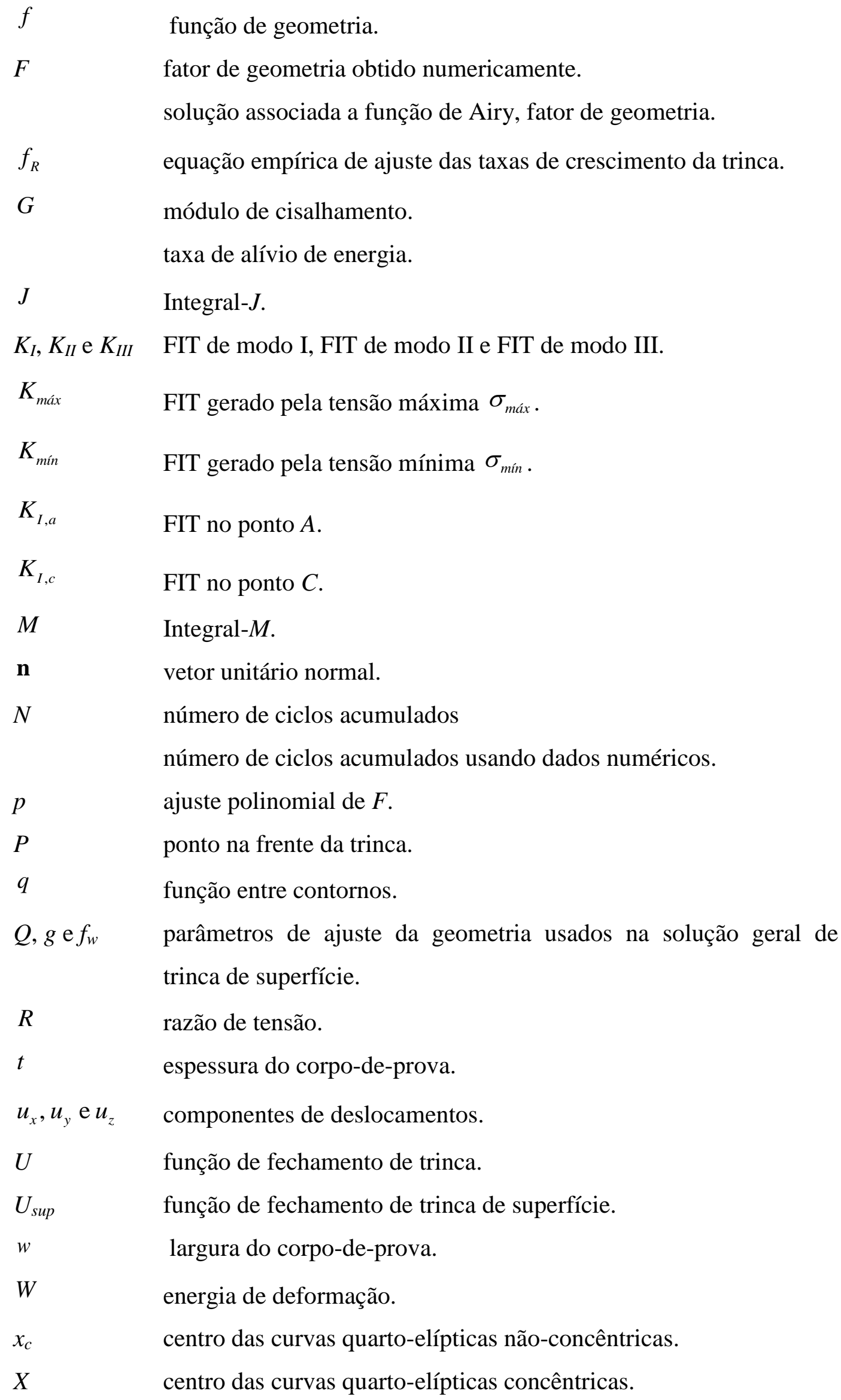




\section{Símbolos gregos}

$\beta \quad$ parâmetro adicional empírico usado nas equações $f_{R}$.

$\delta_{1 j} \quad$ delta Kronecker.

$\boldsymbol{\varepsilon} \quad$ tensor de deformação infinitesimal.

$\phi \quad$ ângulo das coordenadas do ponto na frente da trinca de canto.

$\Phi(r, \theta) \quad$ função arbitrária.

$\Gamma \quad$ contorno de integração.

$v \quad$ coeficiente de Poisson.

$\sigma \quad$ tensão máxima nas superfície da placa ocasionadas por $\Delta P$.

$\sigma_{x x}, \sigma_{y y}, \sigma_{x y}$

$\sigma_{x z}, \sigma_{y z}$ e $\sigma_{z z} \quad$ componentes de tensões.

$\sigma_{\text {máx }} \quad$ tensão máxima ocasionada pelo carregamento cíclico aplicado remotamente.

$\sigma_{\text {min }} \quad$ tensão mínima ocasionada pelo carregamento cíclico aplicado remotamente.

$\Delta \sigma \quad$ faixa de tensão cíclica entre os extremos $\sigma_{\text {min }}$ e $\sigma_{\text {máx }}$. 
Nossos sonhos só podem ser realizados por nós mesmos.

Daisaku Ikeda 